Ann. Biol. anim. Bioch. Biophys., I972, 12 (2), 233-24I.

\title{
SÉGRÉTION PANGRÉATIQUE SUR PORC FISTULÉ ADAPTATION A LA TENEUR EN PROTÉINES DU RÉGIME
}

\author{
T. CORRING et R. SAUCIER (') \\ avec la collaboration technique de Anne-Marie Gueugneau et G. Dunand \\ Station de Recherches sur l'Élevage des Porcs, \\ Centre national de Recherches zootechniques, I. N.R. A., \\ 78 - Jouy-en-Josas
}

\section{RÉSUMÉ}

Plusieurs travaux, réalisés principalement chez le Rat et à partir d'homogénats tissulaires, ont montré que le pancréas exocrine adapte son équipement enzymatique à la compositon du régime alimentaire. Ainsi Desnuelle et al. (1962), Bučco et al. (r968) ont mis en évidence l'adaptation spécifique des enzymes pancréatiques protéolytiques et amylolytiques respectivement à la teneur en protéines et en amidon du régime.

Le phénomène d'adaptation a été testé, ici, selon une méthode originale qui consiste à utiliser le Porc muni d'une fistule pancréatique permanente selon la technique décrite par CorRING et al. (1972). 3 porcs mâles, de race Large White, et de poids vif moyen de $40 \mathrm{~kg}$ ont été soumis, durant quatre périodes de 7 jours, aux régimes successifs suivants : o, 30, 10 et $40 \mathrm{p}$. roo de matières azotées totales. Sur le suc pancréatique recueilli en continu ont été dosées les protéines totales et les activités enzymatiques de la trypsine, chymotrypsine et amylase.

Les résultats mettent en évidence les deux phénomènes suivants :

I La chymotrypsine s'adapte à la teneur en protéines du régime et les différences des activités enregistrées avec les régimes à haute et faible teneur en protéines sont importantes.

Par ailleurs, la réponse de la chymotrypsine se produit régulièrement 48 heures après chaque changement de régime. Les variations des activités enzymatiques de la trypsine et de l'amylase sont relativement peu importantes.

$2^{\circ}$ D'une façon générale, la forte teneur en protéines du régime alimentaire stimule la synthèse protéique totale du pancréas exocrine.

\section{IN'TRODUC'TION}

L a travail présenté a pour but de tester l'adaptation de deux enzymes protéolytiques du pancréas du Porc, la chymotrypsine et la trypsine, à la teneur en protéines du régime alimentaire.

(1) Département de Biologie ; Faculté des Sciences; Université de Sherbrooke, Canada. 
Ce phénomène de l'adaptation de l'équipement enzymatique du pancréas exocrine à la composition du régime alimentaire pressenti par PAVLOV (19I3), a réellement été abordé en I942 par Grossman et al. Par la suite, de nombreux travaux ont été effectués dans le même but et, parmi les plus importants, il faut citer ceux de DESNUELI et ses collaborateurs dont les résultats sont depuis I 962 considérés comme classiques. D'une façon générale, ils établissent qu'un régime à prédominance glucidique ou protidique entraîne une augmentation des activités enzymatiques de l'amylase ou des enzymes protéolytiques et particulièrement de la chymotrypsine.

Ces études ont été réalisées en soumettant les animaux à leur régime durant une période plus ou moins longue ( 2 jours à plusieurs semaines) et ce n'est qu'au terme de cette période, après abattage de l'animal, que l'on a constaté les diverses variations des activités enzymatiques. Cette technique ne peut permettre de suivre sur le même animal, donc d'éviter les grandes variations individuelles, l'évolution enzymatique du pancréas exocrine, notamment lors du changement de régime.

C'est la raison pour laquelle nous avons cherché à mener notre étude sur le Porc, espèce peu utilisée dans ce domaine, muni d'une fistule permanente du pancréas exocrine.

\section{MATÉRIEL ET MÉTHODES}

\section{Animaux}

3 porcs mâles castrés, de race Large White et d'un poids vif moyen de $40 \mathrm{~kg}$ (âgés de $\mathrm{I}$ ro jours environ) en début d'expérimentation, ont été utilisés.

Durant les 8 jours qui ont précédé la fistulation, ils ont été habitués à la cage, ainsi qu'aux diverses conditions expérimentales (distribution des repas, mode de présentation de l'aliment, etc.).

La technique de fistulation consiste à dériver totalement la sécrétion pancréatique à l'extérieur de l'animal, a fin d'en mesurer le volume sécrété et de doser l'activité des enzymes présentes. Après échantillonnage, le suc est réintroduit dans le duodénum. La description de cette technique, ainsi que les conditions d'utilisation des fistules pancréatique et duodénale sont décrites dans un précédent travail (CorRing et al., 1972).

\section{Régimes expérimentaux}

Les animaux ne sont pas placés en expérience durant les 8 jours qui suivent la fistulation, compte tenu de l'influence de l'intervention chirugicale sur la sécrétion pancréatique (CoRRING et al., 1972), et l'on assure simplement la réintroduction de la quantité totale du suc recueilli. Au cours de cette période, ils sont nourris d'un régime équilibré à $16 \mathrm{p}$. Ioo de protéines.

L'expérimentation est effectuée sur 4 périodes de 7 jours en moyenne, au cours desquelles les porcs reçoivent successivement et ad libitum des régimes à o, 3o, 1o et $4^{\circ} \mathrm{p}$. Ioo de protéines, dont la composition est donnée dans le tableau $\mathrm{I}$.

L'aliment est distribué à heures fixes en 2 repas par jour, sous forme de farine mélangée à l'eau. Le rapport farine/eau varie selon l'aliment.

\section{Prélèvements et dosages}

Le suc pancréatique est entièrement recueilli à $+4^{\circ} \mathrm{C}$ en continu et son volume mesuré. Trois prélèvements de $20 \mathrm{ml}$ environ sont effectués par 24 heures : en début de journée, 5 heures après le repas du matin et $\mathrm{I}$ heure après le repas du soir. Cet échantillonnage permet de disposer d'un suc dont la teneur en protéines enzymatiques est variable (Corring et al., 1972). Compte tenu des conditions de récolte (température, $\mathrm{pH}$...), les pro-enzymes proéolytiques ne sont pas 
activés spontanément, et garantissent ainsi le maintien des différentes activités au cours du stockage. Le reste du suc pancréatique est réintroduit par la fistule duodénale au cours de la journée, après réchauffement à $3^{\circ} \mathrm{C}$.

TABLEAU I

Composition des régimes

\begin{tabular}{|c|c|c|c|c|}
\hline \multirow{2}{*}{ Matières azotées } & \multicolumn{4}{|c|}{ Pour $100 \mathrm{~g}$ d'aliment } \\
\hline & $0 \%$ & $10 \%$ & $30 \%$ & $40 \%$ \\
\hline Farine de hareng $\ldots \ldots \ldots$ & - & 13,2 & 39,5 & 52,7 \\
\hline Amidon de mais ....... & 81 & 67,8 & 41,5 & 28,3 \\
\hline Huile d'arachide $\ldots \ldots$. & 3 & 3 & 3 & 3 \\
\hline Cellulose colmacel $\ldots \ldots$ & 12 & 12 & 12 & 12 \\
\hline Mélange minéral $\ldots$. & 3 & 3 & 3 & 3 \\
\hline Mélange vitaminique... & 1 & 1 & 1 & 1 \\
\hline
\end{tabular}

Sur chaque échantillon, on procède à la détermination des protéines totales, de l'activité enzymatique de la chymotrypsine et de la trypsine après activation des précurseurs (CORRING et al., 1972), selon les méthodes utilisées par Corring et Aumaitre (1970).

On effectue également le dosage de l'activité $\alpha$-amylasique selon la méthode de METAIS (I 968 ) sur le sérum que nous avons modifiée en raison des fortes activités rencontrées dans le suc pancréatique. En particulier, toutes les dilutions se font dans $\mathrm{NaCl}$ o,9 p. Ioo et la quantité de substrat a été augmentée ainsi que la concentration de l'acide chlorhydrique ( $\mathrm{I}$ contre $o, \mathrm{I} N$ ). Le substrat est l'amidon soluble de Merck ( $\mathrm{n}^{\circ}$ I 252), préparé à I $p$. Ioo dans un tampon phosphate $\mathrm{pH} 7, \mathbf{1 5}$. L'emploi d'autres amidons a été moins satisfaisant. La solution d'amidon préparée se conserve parfaitement à la température ambiante durant 3 semaines au moins. L'unité déterminée selon cette méthode représente l'unité d'activité amylasique contenue dans $\mathrm{I}, \mathrm{O} \mathrm{ml}$ de suc pancréatique et qui hydrolyse $\mathrm{I}, \mathrm{O} \mathrm{mg}$ d'amidon soluble Merk, durant $3^{\circ} \mathrm{mn}$ à $37^{\circ} \mathrm{C}$.

\section{RÉSULTATS}

La sécrétion enzymatique est exprimée en activités totales par 24 heures et en activités spécifiques moyennes par mg de protéines, par 24 heures.

Un premier résultat se rapporte au développement pondéral des animaux expérimentaux. Ainsi, nous avons enregistré, pour les trois porcs, une croissance moyenne quotidienne de 300,350 et $400 \mathrm{~g}$.

En ce qui concerne l'évolution des différentes activités enzymatiques en fonction du régime ingéré, les réponses obtenues pour les trois porcs sont identiques (tab1. 2) et nous rapporterons les données obtenues à partir de l'un d'entre eux (porc no $\mathrm{I}$ ).

\section{Consommation alimentaire}

Lorsque l'on examine les diagrammes des consommations reportés sur les figures I et 2, deux observations principales peuvent être faites :

Io Au cours de la période de consommation du régime protéiprive, la quantité 
d'aliment ingéré au début est supérieure à $\mathrm{I} 000 \mathrm{~g}$ par jour et diminue très rapidement jusque vers $300 \mathrm{~g}$ par jour.

$2^{\circ}$ Le passage à 1 'aliment contenant $30 \mathrm{p}$. Ioo de protéines s'est immédiatement traduit par une légère augmentation de la consommation (400-450 g/jour) mais les quantités ingérées n'ont pas augmenté par la suite, et les 2 derniers jours de cette seconde période nous avons noté un refus total de consommation. Enfin, le régime à Io $\mathrm{p}$. Ioo de protéines a bien été accepté par les animaux et la consommation a été régulière. Il en a été de même pour le régime à $40 \mathrm{p}$. Ioo de protéines au cours des seuls 5 jours de cette dernière période.

\section{TABLEAU 2}

Activités enzymatiques spécifiques (activités/mg $\mathbf{P t}_{\mathrm{o}}$ ) et protéines totales par ml de suc pancréatique recueilli

Valeurs moyennes quotidiennes obtenues 5 jours après le changement de régime

\begin{tabular}{|c|c|c|c|c|c|}
\hline Régime & Porc $n^{0}$ & $0 \%$ MAT & $30 \% \mathrm{MAT}$ & $10 \% \mathrm{MAT}$ & $40 \% \operatorname{MAT}\left({ }^{1}\right)$ \\
\hline $\begin{array}{l}\text { Protéines en } \gamma / \mathrm{ml} \\
\text { suc pancréatique }\end{array}$ & $\begin{array}{l}1 \\
2 \\
3\end{array}$ & $\begin{array}{l}1000 \\
2400 \\
1900\end{array}$ & $\begin{array}{l}1000 \\
2500 \\
2000\end{array}$ & $\begin{array}{l}1200 \\
2600 \\
2000\end{array}$ & $\begin{array}{c}1300 \\
- \\
-\end{array}$ \\
\hline $\begin{array}{l}\text { Chymotrypsine } \\
\mu \text { moles ATEE/mn }\end{array}$ & $\begin{array}{l}1 \\
2 \\
3\end{array}$ & $\begin{array}{l}20 \\
17 \\
21\end{array}$ & $\begin{array}{l}48 \\
40 \\
52\end{array}$ & $\begin{array}{l}20 \\
18 \\
20\end{array}$ & ${ }^{36}$ \\
\hline $\begin{array}{l}\text { Trypsine } \\
\mu \text { mole BAEE/mn }\end{array}$ & $\begin{array}{l}1 \\
2 \\
3\end{array}$ & $\begin{array}{l}4,5 \\
3,5 \\
4,5\end{array}$ & $\begin{array}{l}6 \\
5,0 \\
5,7\end{array}$ & $\begin{array}{l}4,5 \\
4,0 \\
4,8\end{array}$ & $-{ }^{6}$ \\
\hline $\begin{array}{l}\text { Amylase } \\
\left.\text { unités ( }{ }^{2}\right)\end{array}$ & $\begin{array}{l}1 \\
2 \\
3\end{array}$ & $\begin{array}{l}2000 \\
2400 \\
2920\end{array}$ & $\begin{array}{l}3500 \\
3710 \\
4200\end{array}$ & $\begin{array}{l}2500 \\
2900 \\
3000\end{array}$ & $\begin{array}{c}3000 \\
- \\
-\end{array}$ \\
\hline
\end{tabular}

(1) Valeurs obtenues le $4^{\mathrm{e}}$ jour de cette période.

(2) Unités d'activités amylasique contenues dans $1,0 \mathrm{ml}$ de suc pancréatique et qui hydrolysent 1,0 mg d'amidon soluble Merck, durant $30 \mathrm{mn}$ à $37^{\circ} \mathrm{C}$.

\section{Activités spécifiques moyennes}

Sur 1a figure I, nous avons enregistré l'évolution des activités enzymatiques spécifiques moyennes ainsi que celle de la concentration en protéines totales du suc par 24 heures. Nous avons fait également figurer la quantité d'aliment consommé par jour.

Ainsi, la concentration en protéines du suc pancréatique recueilli a pratiquement très peu varié durant toute l'expérimentation.

En ce qui concerne les enzymes protéolytiques, les variations de l'activité chymotrypsique sont très importantes et suivent celles de la teneur en protéines du régime. Les variations de l'activité trypsique sont relativement faibles. 


\section{Activités enzymatiques totales}

Sur la figure 2, nous avons tracé l'évolution des activités enzymatiques totales de la chymotrypsine, trypsine et amylase ainsi que celle des protéines totales, par 24 heures et en fonction du temps.

Sauf en ce qui concerne la dernière période expérimentale, au cours de laquelle l'ensemble des valeurs augmente de façon importante, nous pouvons noter que la chymotrypsine et l'amylase subissent des variations plus importantes que celles de la trypsine et des protéines.

Cependant, l'expression de ces activités tient compte principalement du volume sécrété par jour. Celui-ci a été faible en début d'expérimentation (700 $\mathrm{ml})$, puis a atteint une valeur moyenne à partir du $4^{\mathrm{e}}$ jour de l'ordre de $\mathrm{I} 800 \mathrm{ml} \pm 300$ jusqu'à la fin de l'étude.

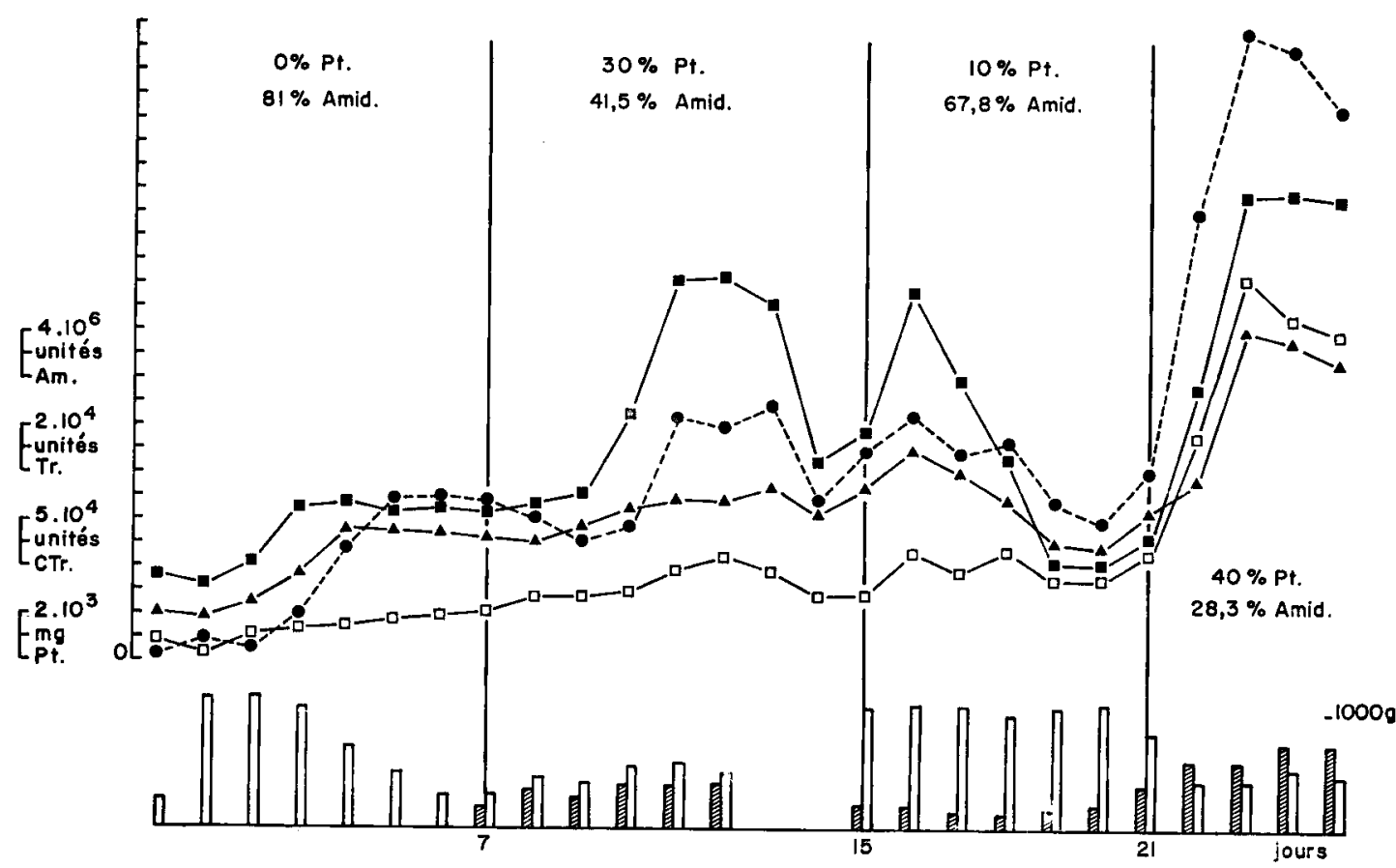

FIG. 2. - Activités enzymatiques totales et protéines totales par 24 heures de la sécrétion pancréatique
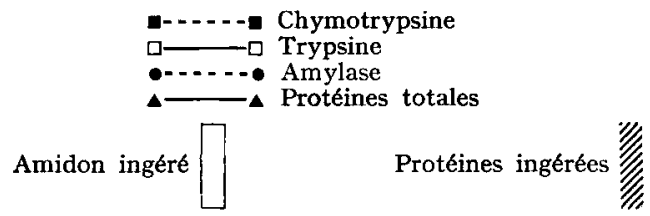

La chymotrypsine répond à la variation de la teneur en protéines du régime et l'activité totale de cette enzyme est maximum pour les deux périodes de consommation des régimes riches en matières azotées. Les variations de la trypsine, par 
contre, sont relativement nulles et nous notons simplement une augmentation régulière de son activité. Cette évolution est à rapprocher de celle de la quantité totale des protéines du suc recueilli par 24 heures.

Enfin, l'activité de l'amylase, avec des amplitudes plus faibles, suit celle de la chymotrypsine.

Par ailleurs, nous retrouvons l'influence du défaut de consommation à la fin de la seconde période et il se traduit par une diminution nette des différentes valeurs ainsi que par une chute du volume de suc recueilli.

\section{DISCUSSION}

Au cours d'un précédent travail (CoRRING et al., I972) sur l'utilisation de porcs fistulés du pancréas exocrine, nous avons insisté sur l'état physiologique des animaux. Dans la mesure où 1'on est amené à utiliser les données obtenues avec des animaux ainsi préparés, un porc dont la croissance est perturbée ne peut être considéré comme offrant toutes garanties quant à la validité des résultats.

Compte tenu de l'influence possible de l'intervention chirurgicale et des régimes expérimentaux, la croissance des porcs utilisés dans ce travail peut être jugée satisfaisante.

D'autre part, le fait que la concentration en protéines du suc pancréatique soit demeurée constante au cours de l'expérimentation montre que les diverses variations enzymatiques peuvent être considérées comme la conséquence de l'influence d'un facteur agissant spécifiquement sur telle ou telle enzyme. En effet, une variation de la synthèse protéique entraînerait une modification de la concentration en protéines du suc et également, de ce fait, une variation des activités enzymatiques, ce qui ne permettrait plus de pouvoir définir l'effet possible d'un facteur tel que la teneur en protéines sur la sécrétion spécifique des enzymes protéolytiques.

De l'ensemble des résultats concernant l'évolution des activités enzymatiques, il ressort que l'activité chymotrypsique varie fortement en fonction des divers régimes ingérés, alors que la trypsine semble être beaucoup moins sensible. Cela confirme que la chymotrypsine répond à toute variation de la composition en protéines alimentaires ainsi que l'avaient montré GRossman et al. (1942) et REBOUD et al. (r966). Cette variation se fait dans le sens d'une adaptation puisque les valeurs de l'activité enzymatique sont les plus élevées pour l'ingestion de protéines alimentaires la plus forte. Comme l'ont montré BEN ABDELJLIL et DESNuELLE (Ig64) l'adaptation est complète dans les 5 à 7 jours qui suivent le changement de régime. Nous avons montré, de plus, que le temps de réponse de la chymotrypsine est de 48 heures.

Si l'on compare ces données à celles observées chez les microorganismes où la réponse survient dans 1'heure qui suit la modification alimentaire, il apparaît que le processus biologique mis en œuvre chez les mammifères dans ce phénomène d'adaptation soit très complexe. Nos résultats confirment également ceux obtenus chez le Rat par LEMIRE et IBER (I956) et LE-TKHAN-UJEN (I969) qui ont mis en évidence la diminution de 1'activité protéolytique du pancréas avec un régime déficient en protéines ou à bas niveau en matières azotées. 
Un résultat concerne la diminution de l'activité spécifique de la chymotrypsine au cours de la période de consommation de l'aliment renfermant 30 p. roo de protéines. Si le refus d'alimentation de la part de l'animal explique aisément la même diminution des activités totales, car il entraîne une chute du volume de suc excrété, il ne peut être exploité ici, la concentration en protéines du suc étant demeurée constante. Il convient de rappeler que, si l'activité spécifique de la chymotrypsine a diminué, un équilibre a été atteint pour des valeurs analogues à celles obtenues avec le régime préexpérimental à $\mathrm{I} 6 \mathrm{p}$. Ioo de protéines. Il semble donc, qu'après une période de consommation d'un aliment protéiprive, le pancréas du porc recevant une alimentation riche en protéines réponde dans les 2 jours en augmentant très fortement, et durant quelques jours, sa sécrétion protéolytique. Un tel résultat a été mis en évidence par IMONDI et BIRD (I967) sur le Poulet. Ces auteurs ont montré, de plus, que l'hyperstimulation s'accompagne d'une hyperplasie. La période de consommation du régime à $40 \mathrm{p}$. Ioo de protéines n'a pu être poursuivie assez longtemps pour constater un même phénomène après l'ingestion de l'aliment à Io $\mathrm{p}$. IOO de matières azotées.

Si l'on considère maintenant l'activité amylasique et les variations mises en évidence en fonction des régimes, 1'évolution des valeurs ne souligne aucune adaptation à la teneur en amidon. En effet, la composition des régimes (tabl. I) faisant apparaître une variation de cette teneur d'un régime à l'autre, on pouvait supposer a priori observer l'adaptation de l'amylase telle que l'ont montrée DESNUELLE (I962), BučKo et KOPEC (I968). En fait, nous constatons que les plus hautes valeurs de l'activité amylasique sont obtenues pour les régimes riches en protéines. Les variations de la teneur en protéines alimentaires étant plus importantes que celles de l'amidon, il semble donc que 1'amylase ait été stimulée en tant que protéine et non pas en tant qu'enzyme hydrolysant les glucides. Un tel résultat avait été mis en évidence par SNOOK et MEYER sur le Rat (I964).

En conclusion, cette étude sur le porc muni d'une fistule pancréatique a permis de confirmer l'adaptation des enzymes protéolytiques et notamment de la chymotrypsine à la teneur en protéines du régime alimentaire. De plus, un régime riche en protéines stimule la synthèse protéique totale du pancréas.

Reçu pour publication en décembre $197 \mathrm{r}$.

\section{SUMMARY}

\section{PANCREATIC SECRETION OF THE FISTULATED PIG.} ADAPTATION TO PROTEIN CONTENT IN THE DIET

Several studies mostly done on rats and using tissue homogenates have shown that the exocrine pancreas adapts its enzymatic system to diet composition. Desnuelle et al. (I96z) and Bucko et al. (1968) demonstrated that proteolytic and amylolytic pancreatic enzymes specifically adapted to protein and starch content in the diet.

The adaptation phenomenon is tested here by an original method using a pig with a permanent pancreatic fistula according to the technique described by CoRRING et al. (1972). For four 7-day periods, 3 Large-White male pigs, weighing an average of $40 \mathrm{~kg}$, received the following successive diets : o-30-10 and $40 \mathrm{p}$. Ioo total proteins. Total proteins and typsine, chymotrypsine and amylase enzymatic activities were determined from the pancreatic sugar collected. 
The results show :

- The chymotrypsine adapts to protein content in the diet and the differences of activities recorded for high and low protein diets are great. Moreover, chymotrypsine response occurs regularly 48 hours after each change in diet. Variations of trypsine and amylase enzymatic activities are relatively insignificant.

- In a general way, high protein diet stimulates total protein synthesis in the exocrine pancreas.

\section{RÉFÉRENCES BIBLIOGRAPHIQUES}

Ben Abdeljlil A., Desnuelle P., 1964. Sur l'adaptation des enzymes exocrines du pancréas à la composition du régime. Biochim. Biophys. Acta 81, 136-I49.

BučKo A., Kopec Z., 1968. Adaptation of enzymes activity of the Rat pancreas on altered food intake. Nutritio et dieta, 10, 276-287.

Corring T., Aumaitre A., Ig7o. Effet du traitement thermique du tourteau de soja sur la variation de l'équipement enzymatique du pancréas exocrine et les performances pondérales du Rat après sevrage. Ann. Biol. anim. Bioch. Biophys., 10, 443-457.

Corring T., Aumaitre A., RERat A., 1972. Fistulation permanente du pancréas exocrine chez le Porc. Application : réponse de la sécrétion pancréatique au repas. Ann. Biol. anim. Bioch. Biophys. 12 IO9-I24.

Desnuelle P., Reboud J.-P., Ben Abdeljlil A., I962. Influence of the composition of the diet on the enzyme content of Rat pancreas, in Ciba Foundation Symposium on the exocrine Pancreas, Churchill (London), p. 90.

Grossman M. I., Greengard H., Ivy C. A., I942. The effect of dietary composition on pancreatic enzymes. Amer. J. Physiol. 138, 676-682.

ImONDI A. R., BIRD F. H., I967. Effects of dietary protein level on growth and proteolytic activity of the avian pancreas. J. Nutr. 91, $421-428$.

Lemire S., Iber F. L., 1966. Pancreatic secretion in rats with protein malnutrition. Johns Hopkins Med. J., 120, $2 \mathrm{I}-25$.

Le-Tkhan-UjEN, 1969. Active adaptability of the pancreas to low-protein diet. Vop. Pitan. 28, $38-43$.

Metais P., Bieth J., Ig68. Détermination de l' $\alpha$-amylase par une microtechnique. Ann. Biol. Clin. $26(I-2), 133-142$.

Pavlov I. P., I913. The work of the digestive glands. Charles Griffin ed. London.

Reboud J.-P., Marchis-Mouren G., Pasero L., Cizzone A., Desnuelle P., ig66. Adaptation de la vitesse de biosynthèse de l'amylase pancréatique et du chymotrypsinogène à des régimes riches en amidon ou en protéines. Biochim. Biophys. Acta, 17, 351-367.

Snook J. T., Meyer J. H., 1964. Response of digestive enzymes to dietary protein. J. Nutr. 82, 409-4I4. 